\title{
Flow Classification and Its Application to Fluid Processing
}

\author{
YASUYA NAKAYAMA*, and Toshihisa KAJIWARA \\ Department of Chemical Engineering, Kyushu University, Fukuoka 819-0395, Japan
}

\begin{abstract}
Mathematically, the problem of flow field classification can be analyzed by the eigenanalysis of the deformation-rate tensor; however, such analysis technique have not been commonly applied in fluid processing. We derive a simplified objective flow classification scheme based on the invariants of the strain-rate tensor and the vorticity tensor. Multiaxiality of flow, which is related to the type of elongation, and converging/bifurcating flow, is characterized by the strain-rate tensor, while rotation contribution that protects fluid element from stretching is characterized by the relative intensity of an objective vorticity to the strain-rate. The spatial distributions of flow classification quantities offer an essential tool in understanding the flow pattern structure, and therefore can be useful to get insights into the connection between the geometry and the process performance.
\end{abstract}

\section{Introduction}

In fluid processes, such as transport, mixing, reaction, and heat transfer, flow pattern is of essential importance to the process performance. Flow pattern or topology is largely controlled by the channel geometry and/or driving elements as well as the operation conditions such as flow rate. Analyzing the flow pattern should be useful for understanding fluid processes in connection with the different geometric structures of channels. In real flow of interest, local flow pattern changes from one spatial point to another, and is not well-defined rheometic flows such as simple shear or simple elongation used in rheological characterization of fluid. In such situations, flow classification scheme of non-rheometric flow is required.

Local flow pattern or topology is determined by the deformation-rate tensor; therefore, the problem of flow field classification can be analyzed by eigenanalysis of the deformation-rate tensor (Tanner and Huilgol, 1975; Chong et al., 1990). However, such analysis technique have not been commonly applied in fluid processing, partly because since this analysis involves the flow topology, intensity, and directions, physical interpretation is not straightforward in real flow fields. It would be useful to be able to calculate quantities that identify the flow topology irrespective of the flow intensity and directions for any flow pattern.

In this paper, we formulate a simplified flow classification scheme based on the invariants of the strain-rate tensor and the vorticity tensor. Application of our flow field classification to flow of a non-Newtonian fluid in melt-mixing in twin-screw extrusion demonstrates that the difference in the channel geometry highly affects the distribution of elongational flow (diverging and bifurcating flows).

\section{Flow classification}

Flow pattern at a point in real flow fields is classified into converging, bifurcating, and planar flows. Rheometric elongational flows are special cases of converging and bifurcating flows, while simple shear flow is classified as planar flow. Elongational and shear flows in real flow fields are different from the ones in rheometic flows. For example, whereas the elongation rate is fixed to twice as compression rate in simple uniaxial elongational flow, the ratio between the elongation and compression rates in general elongational flows does not fixed. In other words, elongational flows in general are uneven multiaxial flow. The question is how to characterize flow topology of such nonrheometric flow in real flow fields.

Since the flow pattern or topology is a kinematic property, criterion for flow classification should be based on the kinematic quantities of flow, not on the rheological properties of fluid, such as the parameters in constitutive models of fluids. Such flow classification criterion can be applied to any real flow of complex fluids irrespective of the rheological properties of the fluid. Astarita (1979) discussed the properties that the flow classification criterion should have. First, it should be a local quantity in the sense it is defined from the local flow field rather than the whole flow field. Since the flow topology varies with spatial position (for instance, see Figure 1), local criterion is used to characterize the spatial variation of the flow topology.

* Corresponding author: nakayama@chem-eng.kyushu-u.ac.jp 
Next, the criterion should be objective; it should be invariant under change of reference frame since the flow topology is independent of the frame of reference. Furthermore, when constructing a constitutive equation representing the rheology dependent on the flow topology, the flow classification criterion can be directly included in the constitutive equation. For this application, the criterion must fulfil the objectivity property that is required for the constitutive equation. We derive scalars representing flow topology that satisfies these properties.

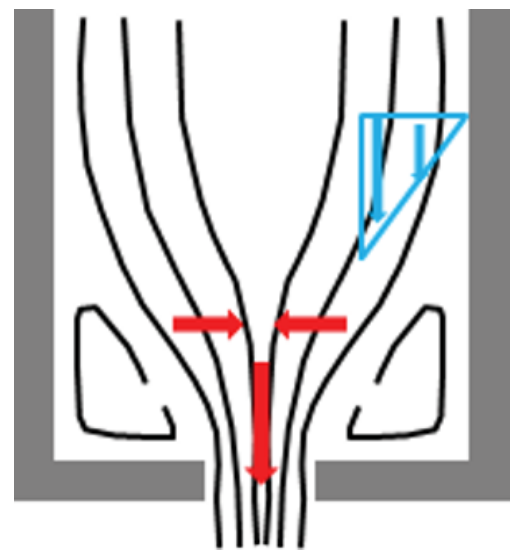

Figure 1. Schematic streamline of flow in a contraction channel. Flow pattern or topology alter with spatial position: flow is close to simple shear near channel wall while close to uniaxial elongational flow around the contracting part. Furthermore, real flow fields is non-rheometric.

Local flow around a point $\mathrm{x}$ is expressed as

$$
\boldsymbol{v}(\boldsymbol{x}+\boldsymbol{r})=\boldsymbol{v}(\boldsymbol{x})+\boldsymbol{r} \cdot \nabla \boldsymbol{v}+\mathrm{O}\left(|r|^{2}\right),
$$

from which we identify $\boldsymbol{v}(\boldsymbol{x})$ is translation and $\nabla \boldsymbol{v}$ determines local flow pattern. The velocity gradient is further decomposed into the strain-rate tensor $\boldsymbol{D}=\left(\nabla \boldsymbol{v}+\nabla \boldsymbol{v}^{t}\right) / 2$ and vorticity tensor $\boldsymbol{\Omega}=\left(\nabla \boldsymbol{v}-\nabla \boldsymbol{v}^{t}\right) / 2$ as $\nabla \boldsymbol{v}=\boldsymbol{D}+\boldsymbol{\Omega}$ where $(.)^{t}$ denotes the transpose. By definition, $\Omega_{i j}=(1 / 2){ }_{i j k} \omega_{k}$ where $\boldsymbol{\omega}=\nabla \times \boldsymbol{v}$, which shows the vorticity tensor represents rigid-body rotation with the angular velocity of $|\omega| / 2$. The multiaxiality of flow is represented by $\boldsymbol{D}$. From this decomposition, we observe that local flow pattern is superposition of multiaxial strain-rate and rotational motion and therefore consider separate characterization of the flow multiaxiality and rotational contribution.

We rederive a scalar of $\boldsymbol{D}$ which characterizes flow multiaxiality, called strain-rate state (Nakayama et al., 2016; Lund and Rogers, 1994; den Toonder et al., 1996), from the geometric viewpoint of the strain-rate tensor. Consider following three invariants of $\boldsymbol{D}: \operatorname{tr} \boldsymbol{D}$, $\boldsymbol{D}: \boldsymbol{D}$, and det $\boldsymbol{D}$. In this paper, we discuss incompressible flow which implies $\operatorname{tr} \boldsymbol{D}=0$. The second-order invariant $\boldsymbol{D}: \boldsymbol{D}=\sum_{i, j} D_{i j}^{2}$ is non-negative, and represents the intensity of $\boldsymbol{D}$. For incompressible flow, the third-order invariant is reduced to $\operatorname{det} \boldsymbol{D}=(1 / 3) \operatorname{tr} \boldsymbol{D}^{3}$. The flow multiaxiality is quantified with $\operatorname{det} \boldsymbol{D}$ as explained below.

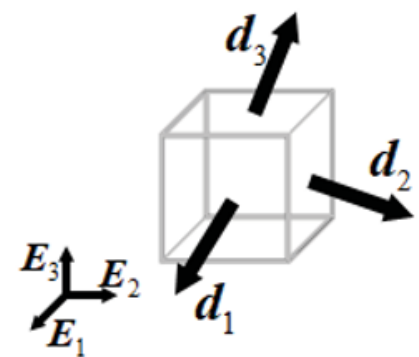

Figure 2. Strain-rate vector $\boldsymbol{d}_{i}=\boldsymbol{E}_{i} \cdot \boldsymbol{D}$ acting on plane normal to the unit vector $\boldsymbol{E}_{i} \cdot(i=1,2,3)$.

The strain-rate tensor consists of three vectors $\boldsymbol{d}_{i}=\boldsymbol{E}_{i} \cdot \boldsymbol{D}(i=1,2,3)$, which represents the strain-rate vector on the plane normal to $\boldsymbol{E}_{i}$, where $\left\{\boldsymbol{E}_{i}\right\}(i=1,2,3)$ is an orthonormal set of basis vectors (Figure 2). For Cartesian basis, the component of a strain-rate vector is $\boldsymbol{d}_{i}=\left(D_{i 1}, D_{i 2}, D_{i 3}\right)$. With $\boldsymbol{d}_{i}$, we have $\operatorname{det} \boldsymbol{D}=\boldsymbol{d}_{1} \cdot\left(\boldsymbol{d}_{2} \times \boldsymbol{d}_{3}\right)$ that is the signed volume of the parallelepiped formed by $\left\{\boldsymbol{d}_{i}\right\}(i=1,2,3)$. This geometric interpretation enables us to relate the multiaxiality of flow and the value of $\operatorname{det} \boldsymbol{D}$. In planar flow, one of the strain-rate vectors vanishes, and thus the parallelepiped collapses, leading to $\operatorname{det} \boldsymbol{D}=0$. By contrast, when all three strain-rate vectors are nonvanishing, $\operatorname{det} \boldsymbol{D}$ has a finite value, which indicates a triaxial flow and represents a general kind of elongational flow including rheometric and non-rheometric ones. Uniaxial elongation consists of compression in two directions, $\boldsymbol{d}_{i}, \boldsymbol{d}_{j}<0$ for $i \neq j$, and elongation in the other $k$ direction, $\boldsymbol{d}_{k}>0$, leading to $\operatorname{det} \boldsymbol{D}>0$, which represents a converging flow. On the other hand, biaxial elongational flow consists of $\boldsymbol{d}_{i}, \boldsymbol{d}_{j}>0$ and $\boldsymbol{d}_{k}<0$ leading to $\operatorname{det} \boldsymbol{D}<0$, which represents bifurcating flow. In short, the sign of $\operatorname{det} \boldsymbol{D}$ discriminates flow multiaxiality, which is a toplogical property of flow independent of the flow strength.

The value of $\operatorname{det} \boldsymbol{D}$ is determined by not only the type of multiaxial flow but the intensity of the strain-rate as $\operatorname{det} \boldsymbol{D} \propto(\sqrt{\boldsymbol{D}: \boldsymbol{D}})^{3}$. With proper normalization, we derive a scalar, called the strain-rate state, that characterizes the flow multiaxiality independent of the shear-rate as

$$
s=\frac{3 \sqrt{6} \operatorname{det} \boldsymbol{D}}{(\boldsymbol{D}: \boldsymbol{D})^{3 / 2}},
$$


where the numerical factor is determined to be $s \in[-1,1]$. Since $\mathrm{s}$ is based on the invariants of $\boldsymbol{D}, s$ is objective. The multiaxiality of flow at a spatial point is quantified by the strain-rate state. The strain-rate state has been used to analyze the relation between the channel geometry and non-Newtonian flow (Nakayama et al., 2016, 2018), and discuss the elongational flow distribution in turbulence (Lund and Rogers, 1994; den Toonder et al., 1996).

We illustrate the relation between the flow multiaxiality and the value of s. For this purpose, we only need to see the three principal strain-rates calculated by diagonalizing $\boldsymbol{D}$,

$$
\boldsymbol{D}=\boldsymbol{T} \cdot\left(\begin{array}{ccc}
\dot{\varepsilon}_{1} & 0 & 0 \\
0 & \dot{\varepsilon}_{2} & 0 \\
0 & 0 & \dot{\varepsilon}_{3}
\end{array}\right) \cdot \boldsymbol{T}^{t}
$$

where $\boldsymbol{T}$ is orthonormal matrix. In biaxial elongational flow, $\dot{\varepsilon}_{1}, \dot{\varepsilon}_{2}>0, \dot{\varepsilon}_{3}<0$, leading to $-1 \leq s<0$. Especially in equi-biaxial elongational flow, $\dot{\varepsilon}_{1}=\dot{\varepsilon}_{2}=-\dot{\varepsilon}_{3} / 2$ and $s=-1$. In uniaxial elongational flow, $\dot{\varepsilon}_{1}>0$, $\dot{\varepsilon}_{2}, \dot{\varepsilon}_{3}<0$, leading to $0<s \leq 1$. Especially in simple elongational flow, $-\dot{\varepsilon}_{1} / 2=\dot{\varepsilon}_{2}=\dot{\varepsilon}_{3}$ and $s=1$. In planar flow, $\dot{\varepsilon}_{1}>0, \dot{\varepsilon}_{2}<0, \dot{\varepsilon}_{3}=0$, leading to $s=0$. The value of $s$ depends on the balance of relative intensities of $\dot{\varepsilon}_{1}, \dot{\varepsilon}_{2}$, and.$\dot{\varepsilon}_{3}$.

We add a comment on the complete characterization of the multiaxial flow for better understanding what $s$-value implies. In general, multiaxial flow is described by three properties: the type, the strength which is commonly comcharacterize by elongational/shear rate, and the directions. These three properties are defined with the eigenanalysis of $\boldsymbol{D}$ in the first place. From this viewpoint, $s$ only determines the type of multiaxial flow. The advantage is that spatial variation of the multiaxiality can be directly visualized and analyzed with $s$ since $s$ is a scalar.

Next, we consider the contribution of the vorticity tensor on the multiaxial flow. The vorticity tensor or equivalently the vorticity vector has three invariants: intensity of vorticity, and direction relative to the principal axis of $\boldsymbol{D}$ which is measured with two angles. The vorticity represents the rigid-body rotational motion of fluid elements. This rotational motion itself does not strain the fluid element, but reduce the stretching effect of $\boldsymbol{D}$ by shifting the direction of the fluid element away from the highest stretching direction of $\boldsymbol{D}$. From the flow pattern viewpoint, we concern the relative contribution of $\boldsymbol{\Omega}$ to the multiaxial flow.

We note that $\boldsymbol{\Omega}$ itself is not objective, but the relative rotation rate to that of the principal axes of $\boldsymbol{D}$ is objective (Astarita, 1979). Here, we introduce relative rate-of-rotation tensor according to Astarita (1979) as follows. Let the set of unit eigenvector of $\boldsymbol{D}$ be $\left\{\boldsymbol{e}_{i}\right\}(i=1,2,3)$. The angular velocity vector of $\boldsymbol{e}_{i}$ and the corresponding tensor are defined by

$$
\begin{gathered}
\boldsymbol{w}=\boldsymbol{e}_{i} \times \frac{D \boldsymbol{e}_{i}}{D t}, \\
W_{i j}={ }_{i j k} w_{k} .
\end{gathered}
$$

The relative rate-of-rotation tensor is defined by

$$
\boldsymbol{\Omega}_{r e l}=\boldsymbol{\Omega}-\boldsymbol{W} \text {. }
$$

Objectivity of $\boldsymbol{\Omega}_{\text {rel }}$ is shown as follows. Consider a change of frame by $\boldsymbol{x}^{\prime}=\boldsymbol{Y}(t) \cdot \boldsymbol{x}+\boldsymbol{a}(t)$. Along with this, $\boldsymbol{\Omega}$ and $\boldsymbol{e}_{i}$ are transformed as

$$
\begin{aligned}
& \boldsymbol{\Omega}^{\prime}=\boldsymbol{Y} \cdot \boldsymbol{\Omega} \cdot \boldsymbol{Y}^{t}-\dot{\boldsymbol{Y}} \cdot \boldsymbol{Y}^{t}, \\
& \boldsymbol{e}_{i}{ }^{\prime}=\boldsymbol{Y} \cdot \boldsymbol{e}_{i} .
\end{aligned}
$$

Differentiation of $\boldsymbol{e}_{i}{ }^{\prime}$ is

$$
\begin{aligned}
\frac{D \boldsymbol{e}_{i}{ }^{\prime}}{D t} & =\dot{\boldsymbol{Y}} \cdot \boldsymbol{e}_{i}+\boldsymbol{Y} \cdot \frac{D \boldsymbol{e}_{i}}{D t} \\
& =\dot{\boldsymbol{Y}} \cdot\left(\boldsymbol{Y}^{t} \cdot \boldsymbol{e}_{i}{ }^{\prime}\right)+\boldsymbol{Y} \cdot\left(-\boldsymbol{W} \cdot \boldsymbol{e}_{i}\right) \\
& =\left(\dot{\boldsymbol{Y}} \cdot \boldsymbol{Y}^{t}-\boldsymbol{Y} \cdot \boldsymbol{W} \cdot \boldsymbol{Y}^{t}\right) \cdot \boldsymbol{e}_{i}{ }^{\prime},
\end{aligned}
$$

where $\quad D \boldsymbol{e}_{i} / D t=\boldsymbol{e}_{i} \cdot \boldsymbol{W} \quad$ is used. Since $D \boldsymbol{e}_{i}{ }^{\prime} / D t=\boldsymbol{e}_{i}{ }^{\prime} \cdot \boldsymbol{W}^{\prime}$, the transformation of $\boldsymbol{W}$ is

$$
\boldsymbol{W}^{\prime}=\boldsymbol{Y} \cdot \boldsymbol{W} \cdot \boldsymbol{Y}^{t}-\dot{\boldsymbol{Y}} \cdot \boldsymbol{Y}^{t},
$$

where $\boldsymbol{Y} \cdot \boldsymbol{Y}^{\prime}=\boldsymbol{I}$ is used and $\boldsymbol{I}$ is the unit tensor. Although $\boldsymbol{\Omega}$ and $\boldsymbol{W}$ are not objective, they follow the same transformation rule, which leads to the transformation rule of $\boldsymbol{\Omega}_{r e l}^{\prime}=\boldsymbol{Y} \cdot \boldsymbol{\Omega}_{r e l} \cdot \boldsymbol{Y}^{t}$, showing the objectivity of $\boldsymbol{\Omega}_{\text {rel }}$.

Using $\boldsymbol{D}$ and $\boldsymbol{\Omega}_{\text {rel }}$, Astarita (1979) introduced relative strength of vorticity to strain-rate as $r=\left\|\boldsymbol{\Omega}_{\text {rel }}\right\| /\|\boldsymbol{D}\|$ where $\|\boldsymbol{A}\|=\sqrt{\sum_{i j} A_{i j}^{2}}$ is Frobenius norm of a tensor $\boldsymbol{A}$. The flow is irrotational or straindominated when $r=0$, half rotational as in the simple shear flow when $r=1$, and rigid-body rotation where $r \rightarrow \infty$. The $r$ is a measure of the degree to which the fluid avoids stretching. Using $r$, objective irrotationality is defined by

$$
E=\frac{1}{1+r}=\frac{\|\boldsymbol{D}\|}{\|\boldsymbol{D}\|+\left\|\boldsymbol{\Omega}_{r e l}\right\|} .
$$

In contrast that $r \in[0, \infty), E \in[0,1]$. The irrotationality $E$ is a measure of the strain-dominance of the flow. Especially, the flow is strain-dominated or irrotational when $E=1$, half rotational when $E=1 / 2$, and fully rotational when $E=0$. Simpler definition of irrotationality, where $\boldsymbol{\Omega}$ instead of $\boldsymbol{\Omega}_{\text {rel }}$ is used, have been often used in the literatures to study flow-type distribution (Ottino, 1989; Yang and ManasZloczower, 1992; Yao and Manas-Zloczower, 1998; 
Jongen, 2000; Yao et al., 2001; Connelly and Kokini, 2006).

Two scalars, $S$ and $E$, characterize different aspects of the topology of flow, and are combinedly used to classify flow as depicted in Figure 3. The type of elongational flow is determined by the strain-rate state $S$ while the relative effect of vorticity on the multiaxial flow is determined by the irrotationality $E$. Figure 3 shows that rheometric flows (such as simple shear, simple elongation, equi-biaxial elongation etc.) are special cases in general flow classification.

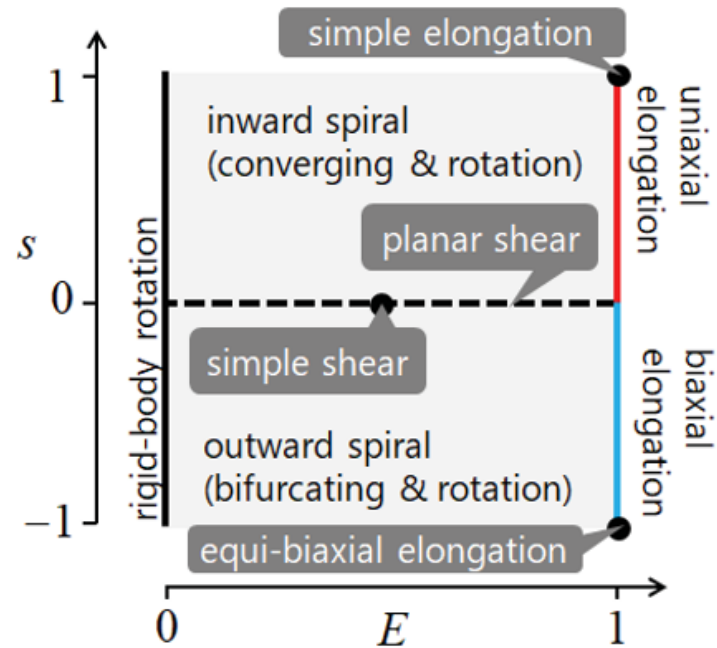

Figure 3. Flow classification with the strain-rate state, $S$, and the

irrotationality, $E$. Multiaxiality of flow is determined with $\mathrm{s}$ which characterizes whether the flow is converging or bifurcating while the relative contribution of vorticity is characterized by E. Rheometric flows characterized by specific values of $\mathrm{s}$ and $\mathrm{E}$ are indicated in $(\mathrm{s}, \mathrm{E})$ plane.

In the past, other methods of flow classification have been discussed. A method based on the invariants of $\nabla \boldsymbol{v}$ that appear in its eigenpolynomial $\lambda^{3}-P \lambda^{2}+Q \lambda-R=0$ has been proposed (Chong et al., 1990), though $\nabla \boldsymbol{v}$ is not objective. For incompressible flow, since $P=\nabla \cdot \boldsymbol{v}=0, Q$ and $R$ are concerned. The second-order invariant, $Q=\left(\boldsymbol{\Omega}: \boldsymbol{\Omega}^{t}-\boldsymbol{D}: \boldsymbol{D}\right) / 2$, gives a qualitative measure of relative intensity of $\boldsymbol{D}$ and $\boldsymbol{\Omega}$ while the third-order invariant, $R=\operatorname{det} \nabla \boldsymbol{v}$, is related to the vortex stretching and flow multiaxiality; however, since $Q$ and $R$ are not normalized, physical interpretation of the values of them is not so obvious.

To determine the dominance of strain-rate, eigenvalue discriminant of $\nabla \boldsymbol{v}$, $\Delta=\left(\operatorname{tr} \nabla v^{2}\right)^{3}-6\left(\operatorname{tr} \nabla v^{3}\right)^{2}$, has been used (Einarsson et al., 2018). Positive $\Delta$ indicates the eigenvalues of $\nabla \boldsymbol{v}$ are all real, namely $\boldsymbol{\Omega}$ is vanishing, while negative $\Delta$ indicates the complex eigenvalues due to finite vorticity. In this case, the sign of $\Delta$ is used to discriminate the fully strain-dominant (fully irrotational) or not; however, the interpretation of the value of $\Delta$ is not clear. Difficulty in interpretation of $Q, R$, and $\Delta$ is because they reflect not only the flow type but also the intensity of $\boldsymbol{D}$ and $\boldsymbol{\Omega}$. By contrast, with $S$ and $E$, only flow topology can be determined regardless of the intensity of $\boldsymbol{D}$ and $\boldsymbol{\Omega}$.

\section{Channel Geometry and Flow Pattern Distribution}

We show how flow pattern is altered by the channel geometry. For flow in co-rotating twin-screw extrusion, we compare flow pattern distribution in different screw element geometries. In extrusion processes, flow is driven by screw rotation, which induces circumferential shear flow, and material-feeding flow rate, which induces pressure flow. Figure 4 shows a configuration of full-flight screw (FS) and neutral kneading block (NKB). Although the shapes of FS and NKB are different, their cross-section is the same due to geometrical constraint in co-rotating twin screws so that they do not contact during the screw rotation.

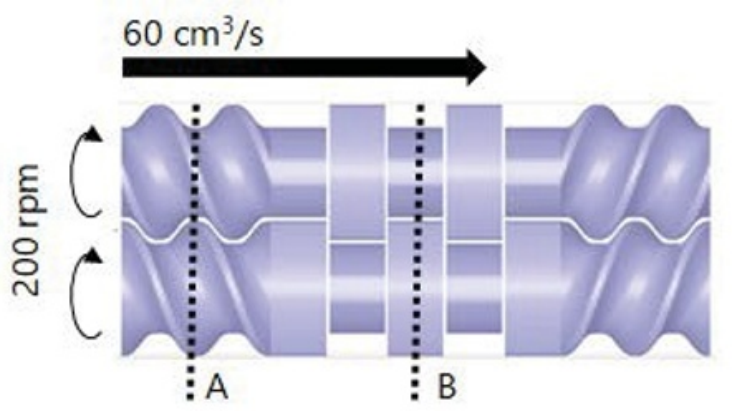

Figure 4. Screw configuration of the melt-mixing zone in a twin-screw extruder with a barrel diameter of 69 $\mathrm{mm}$

We consider the incompressible flow of viscous shear-thinning fluid completely filled in the channel. The constitutive equation is $\boldsymbol{\tau}=2 \eta \boldsymbol{D}$, $\eta=\eta_{0} /\left(1+c\left(\eta_{0} \dot{\gamma}\right)^{n}\right), \quad \eta_{0}=a \exp (b / T)$. The parameters were set to $a=1.7394 \mathrm{~Pa} \cdot \mathrm{s}$, $b=4656.8 \mathrm{~K} \quad, \quad n=0.66941 \quad, \quad$ and $c=1.3575 \times 10^{-3} \mathrm{~Pa}^{-n}$. For comparison, flow of a Newtonian fluid with viscosity $\eta=4000 \mathrm{~Pa} \cdot \mathrm{s}$ was also calculated. Flow is assumed to be pseudo-steady to screw rotation and was solved with finite volume method with a software "R-FLOW"(R-flow Co., Saitama, Japan) under the screw rotation speed of $200 \mathrm{rpm}$ and a flow rate of $60 \mathrm{~cm}^{3} / \mathrm{s}$. The other detail of the numerical calculation is the same as described in Nakayama et al. (2016).

Figure 5 shows shear-rate, irrotationality, and strain-rate state of the shear-thinning fluid flow in crosssections in FS and NKB. Note that a simpler definition of irrotationality $E^{\prime}=\|\boldsymbol{D}\| /(\|\boldsymbol{D}\|+\|\boldsymbol{\Omega}\|)$ is used in 
Figure 5 for convenience. The strain-rate is largest in narrow-gap regions such as tip-barrel and inter-disc clearances, and is rather small in other areas. The shearrate distribution is similar in FS and NKB. Irrotationality distribution reveals that half-rotational flow is seen near wall while irrotational flow develops regions far from wall. This feature is common to FS and NKB. Note that the region of $E^{\prime} \approx 1$ far from the wall is straindominated but the stretching should not be expected to be so strong since the shear-rate there is not large. By contrast, the strain-state distribution differs greatly between FS and NKB. In FS, planar shear flow prevails whereas in NKB bifurcating and converging flow develop at front and backside of the rotating disc, respectively. Figure 5 demonstrates that the strain-rate state distribution reflects the difference between the flow patterns of FS and NKB.
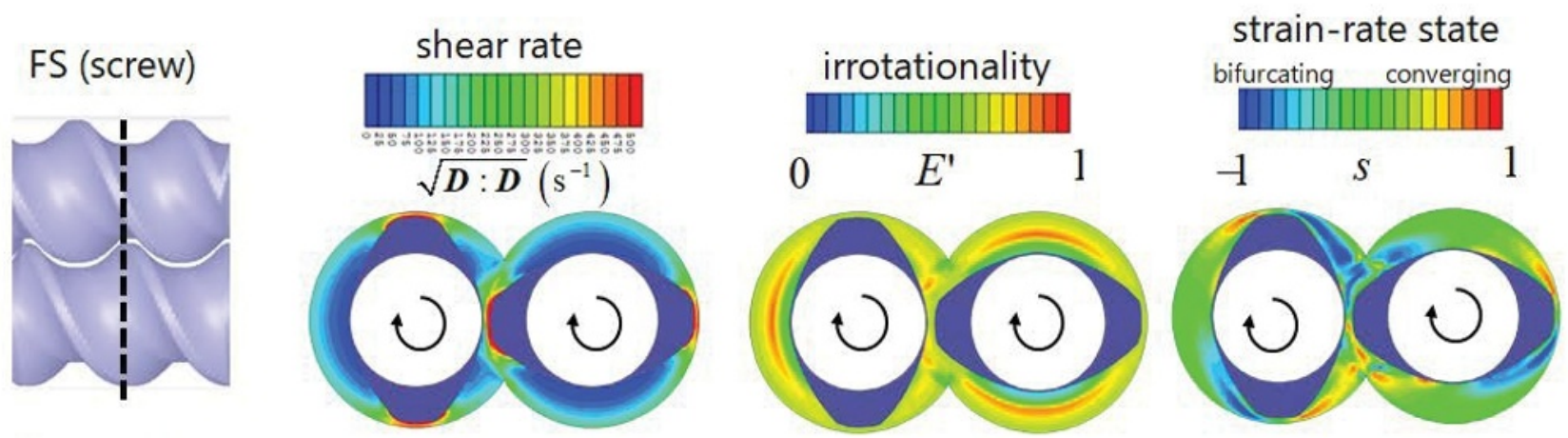

\section{$\mathrm{KB}$ (kneading block)}
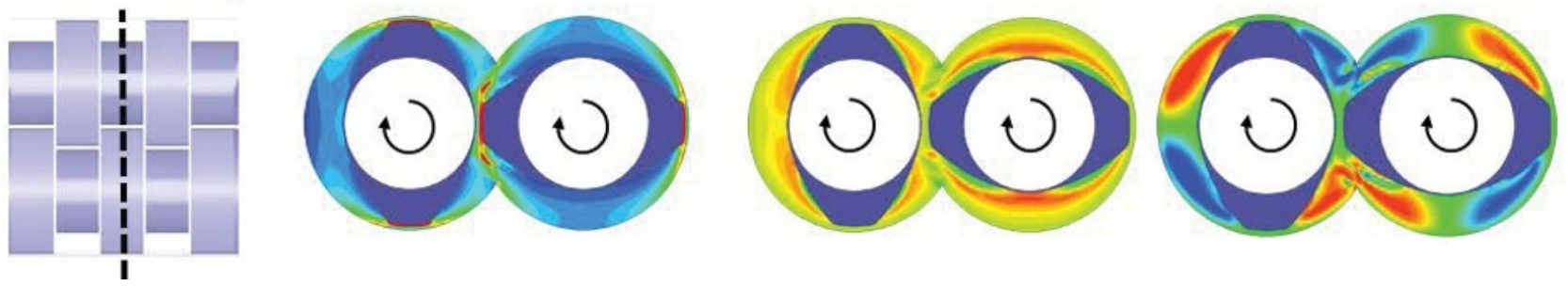

Figure 5. Flow pattern distributions of a shear-thinning fluid flow in cross-sections indicated in Figure 4 that are in conveying full-flight screw and kneading block, respectively. Irrotationality is calculated with $\Omega$ for convenience.
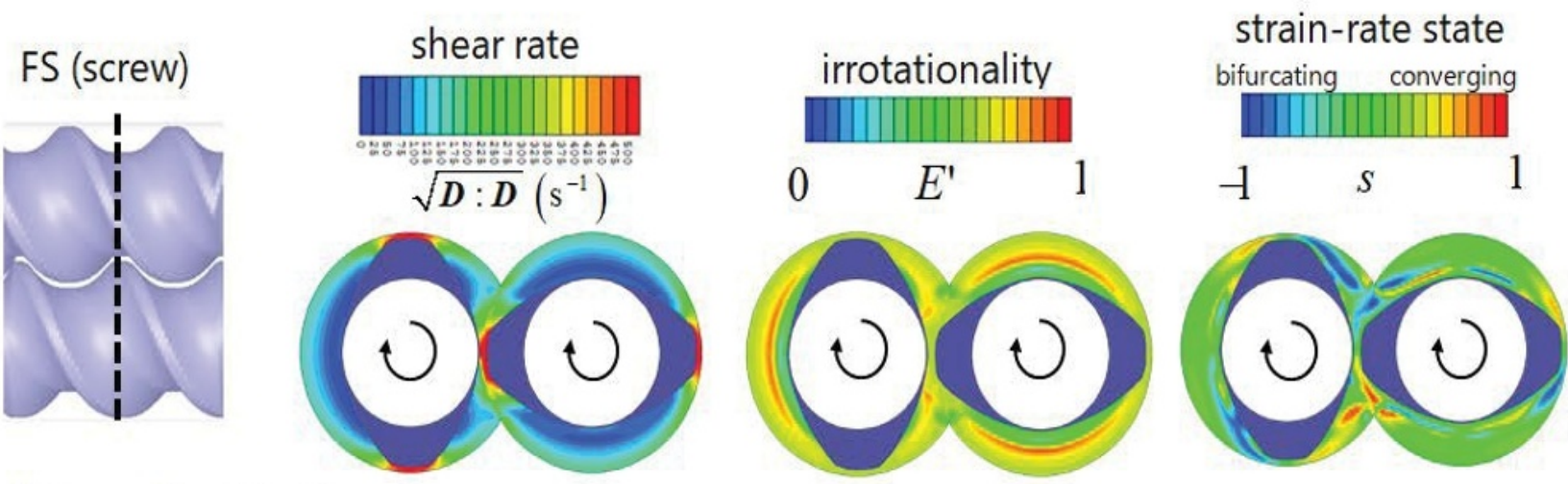

\section{$\mathrm{KB}$ (kneading block)}
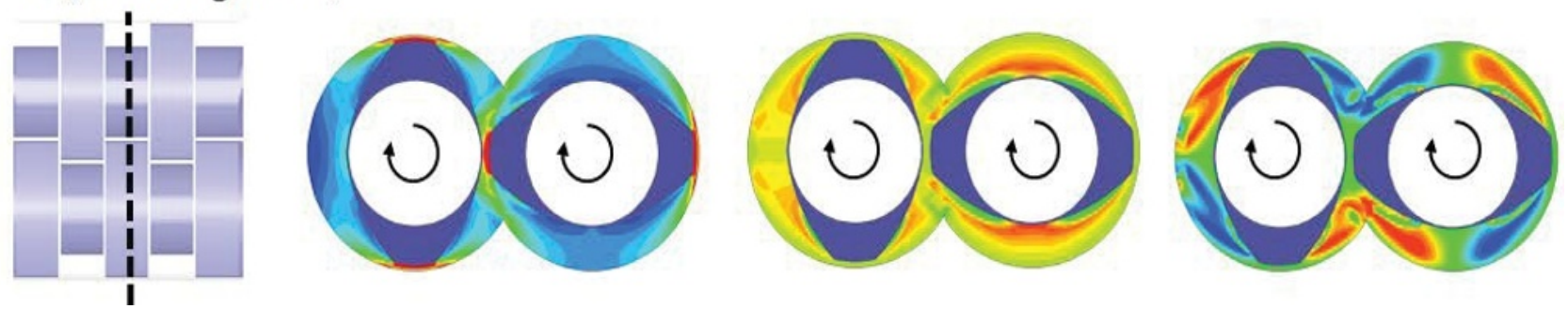

Figure 6. Flow pattern distributions of a Newtonian fluid flow in cross-sections indicated in Figure 4 that are in conveying fullflight screw and kneading block, respectively. Irrotationality is calculated with $\Omega$ for convenience.

Figure 6 shows the same plot as in Figure 5 but for the flow of a Newtonian fluid. Compared to the case of the shear-thinning fluid, Newtonian fluid flow exhibits a slightly thicker boundary layer near the wall; 
nevertheless, there is no significant difference in the flow pattern structures between the shear-thinning and Newtonian fluid cases. This result demonstrates that flow pattern of viscous fluids in the channel shown in Figure 4 is mainly determined by the geometry of the channel.

\section{Conclusions}

We formulated a method of flow field classification using two scalars derived from the strain-rate tensor and the vorticity tensor. The two scalars are the strain-rate state, which measures multiaxiality of flow, and the irrotationality, which measures the strain-dominance of the flow. The method enable us to identify the local flow pattern in non-rheometric flow encountered in real flow and discuss the flow pattern distribution. Since the flow classification method proposed in this manuscript can be applied to flow in different fluid processes, it is expected to be an essential tool for understanding the fluid processes.

\section{Acknowledgements}

The numerical calculations have been partly carried out using the computer facilities at the Research Institute for Information Technology in Kyushu University. This work has been supported by Grants-in-Aid for Scientific Research (JSPS KAKENHI) under Grant No. JP18K03563.

\section{References}

Astarita, G.; "Objective and generally applicable criteria for flow classification," J. Non-Newt. Fluid Mech., 6, 69-76 (1979)

Chong, M. S., A. E. Perry, and B. J. Cantwell; "A General Classification of Three-Dimensional Flow Fields," Phys. Fluids A, 2, 765-777 (1990)

Connelly, R. K. and J. L. Kokini; "3D Numerical Simulation of the Flow of Viscous Newtonian and Shear Thinning Fluids in a Twin Sigma Blade Mixer," $A d v$.

Polym. Technol., 25, 182-194 (2006)

Einarsson, J., M. Yang, and E. S. G. Shaqfeh; "Einstein Viscosity with Fluid Elasticity," Phys. Rev. Fluids, 3, 013301 (2018)

Jongen, T.; "Characterization of Batch Mixers Using Numerical Flow Simulations," AIChE J., 46, 2140-2150 (2000)

Lund, T. S. and M. M. Rogers; "An Improved Measure of Strain State Probability in Turbulent Flows," Phys. Fluids, 6, 1838-1847 (1994)

Nakayama, Y., T. Kajiwara, and T. Masaki; "Strain Mode of General Flow: Characterization and Implications for Flow Pattern Structures," AIChE J., 62, 2563-2569 (2016)
Nakayama, Y., H. Takemitsu, T. Kajiwara, K. Kimura, T. Takeuchi, and H. Tomiyama; "Improving Mixing Characteristics with a Pitched Tip in Kneading Elements in Twin-Screw Extrusion," AIChE J., 64, 1424-1434(2018)

Ottino, J. M.; The Kinematics of Mixing: Stretching, Chaos, and Transport, Cambridge University Press, Cambridge, U.K. (1989)

Tanner, R. I. and R. R. Huilgol; "On a Classification Scheme for Flow Fields," Rheol. Acta, 14, 959-962 (1975)

den Toonder, J. M. J., G. D. C. Kuiken, and F. T. M. Nieuwstadt; "A Criterion for Identifying Strong Flow Regions in Turbulence," Euro. J. mech. B, 15, 735-753 (1996)

Yang, H. H. and I. Manas-Zloczower; "Flow Field Analysis of the Kneading Disc Region in a Co-Rotating Twin Screw Extruder," Polym. Eng. Sci., 32, 14111417 (1992)

Yao, C. H. and I. Manas-Zloczower; "Influence of Design on Dispersive Mixing Performance in an Axial Discharge Continuous Mixer -LCMAX 40," Polym. Eng. Sci., 38, 936-946 (1998)

Yao, W. G., S. Tanifuji, K. Takahashi, and K. Koyama; "Mixing Efficiency in a Pin Mixing Section for SingleScrew Extruders," Polym. Eng. Sci., 41, 908-917 (2001) 